\title{
Dependence of Magnetic Damping on Temperature and Crystal Orientation in Epitaxial $\mathrm{Fe}_{4} \mathrm{~N}$ Thin Films
}

\author{
S. Isogami, M. Tsunoda*, M. Oogane**, A. Sakuma**, and M. Takahashi*** \\ Department of General Education, Fukushima National College of Technology, Iwaki 970-8034, Japan \\ * Department of Electronic Engineering, Tohoku University, Sendai 980-8579, Japan \\ ** Department of Applied Physics, Tohoku University, Sendai 980-8579, Japan \\ *** The New Industry Creation Hatchery Center, Tohoku University, Sendai 980-8579, Japan
}

In-plane and out-of-plane ferromagnetic resonance (FMR) were used to investigate the intrinsic magnetic damping constant $(\alpha)$ in epitaxial $\mathrm{Fe}_{4} \mathrm{~N}$ thin films deposited on $\mathrm{MgO}$ substrates. The dependence of $\alpha$ on temperature was evaluated from room temperature (RT) to $4 \mathrm{~K}$. The external magnetic field (H) of FMR was applied in two directions, i.e., [100] and [110], of the $\mathrm{Fe}_{4} \mathrm{~N}$ lattice. Anisotropic $\alpha$ was observed from RT to $4 \mathrm{~K}$. Moreover, the $\alpha$ for $H / /$ [100] exceeded the $\alpha$ for $H / /$ [110] at $180 \mathrm{~K}$. Numerical calculations of $\alpha$ for bulk $\mathrm{Fe}_{4} \mathrm{~N}$ revealed the same behavior as that in the experiments. The temperature dependence of anisotropic $\alpha$ was explained by the changes in the electronic band structure depending on the directions of magnetization.

Key words: magnetic damping, ferromagnetic resonance, $\mathrm{Fe}_{4} \mathrm{~N}$ thin film

\section{Introduction}

$\mathrm{Fe}_{4} \mathrm{~N}$ thin films with the $\gamma^{\prime}$ phase have attracted attention due to the negative spin polarization of the density of states (DOS). ${ }^{1}$ Using a $\mathrm{Fe}_{4} \mathrm{~N}$ thin film as an FM electrode of the magnetic tunnel junction (MTJ), $\mathrm{Fe}_{4} \mathrm{~N} / \mathrm{MgO} / \mathrm{CoFeB}-\mathrm{MTJ}$, we reported an inverse tunnel magnetoresistive (inverse-TMR) effect and inverse current-induced magnetization switching (inverse-CIMS)..$^{2-5}$ ) One of the advantages of the inverse properties is that highly efficient spin-torque oscillation can be expected in a MTJ with dual $\mathrm{MgO}$ barriers, for example, $\mathrm{CoFeB}$ (pinned layer) / $\mathrm{MgO} / \mathrm{CoFeB}$ (free layer) / $\mathrm{MgO} / \mathrm{Fe}_{4} \mathrm{~N}$ (pinned layer), because spin-torque transfers from both the pinned layers. For application of $\mathrm{Fe}_{4} \mathrm{~N}$ films in spintronics devices, the magnetic damping properties need to be understood, since the critical switching current density in CIMS strongly depends on them.

In recent years, down-sizing of magnetic elements into the several-10s of nanometer range has become indispensable for high areal densities. Nanoscale magnets exclude magnetic domain structures and the resultant anisotropic magnetic damping might be predominant. The anisotropic damping constant of epitaxial films has been reported by some groups. For example, R. Yilgin et al. reported that maximum and minimum "intrinsic" damping constants $(\alpha)$ were observed at room temperature (R.T.) in the hard-axis and the easy-axis of epitaxial $\mathrm{Co}_{2} \mathrm{MnSi}$ films, respectively. ${ }^{6)}$ Other reports have revealed anisotropic $\alpha$ at R.T. in hcp and bcc-Co epitaxial films. ${ }^{7)}$ On the other hand, anisotropy has not been reported in $\mathrm{Fe}$ and fcc-Co epitaxial films. ${ }^{8-10)}$ These reports have led us to consider that not all films have anisotropic $\alpha$, and it is not clear what determines the occurrence of anisotropic $\alpha$.
For the microscopic origins of magnetic damping, several researchers have proposed the spin-orbit interaction, which is the coupling between spin and lattice, and the spin-flip, due to impurities.11-14) Such intrinsic origins become predominant by excluding the thermal fluctuation of spins. Kambersky and Gilmore et al. introduced the electron lifetime $(\tau)$, which can be converted to temperature, and explained the experimental data regarding the temperature (down to $4 \mathrm{~K})$ dependence of $\alpha$ conducted by Bhagat et al.15-17); nevertheless, they did not address the issues of the mechanisms of anisotropic $\alpha$.

We investigated $\alpha$ of "poly"-crystalline $\mathrm{Fe}_{4} \mathrm{~N}$ films in a previous study. ${ }^{18)}$ In contrast, epitaxial $\mathrm{Fe}_{4} \mathrm{~N}$ films, considered an important spintronics material for the future, excited our curiosity based on how its magnetocrystalline anisotropy acts upon anisotropic $\alpha$. The present work was intended to evaluate the temperature dependence of $\alpha$ by means of in-plane and out-of-plane ferromagnetic resonance (FMR), as well as numerical calculations, to promote our understanding of the mechanism of anisotropic $\alpha$.

\section{Experimental and analysis procedure}

100-nm-thick epitaxial $\mathrm{Fe}_{4} \mathrm{~N}$ films were deposited on $\mathrm{MgO}$ substrates using an ultra-high-vacuum magnetron sputtering system. Prior to the deposition, a dry-etching process on the $\mathrm{MgO}$ substrate was performed with an $\mathrm{RF}$ power density of $0.4 \mathrm{~W} / \mathrm{cm}^{2}$. To promote chemical ordering of $\gamma^{\prime}-\mathrm{Fe}_{4} \mathrm{~N}$ films, in situ IR thermal treatment was performed at $300{ }^{\circ} \mathrm{C}$ for $15 \mathrm{~min}$ immediately after deposition of the $\mathrm{Fe}_{4} \mathrm{~N}$ film. After cooling the film to R.T. in 30 min, a 1-nm-thick Ta cap layer was deposited on the $\mathrm{Fe}_{4} \mathrm{~N}$ film surface. The 

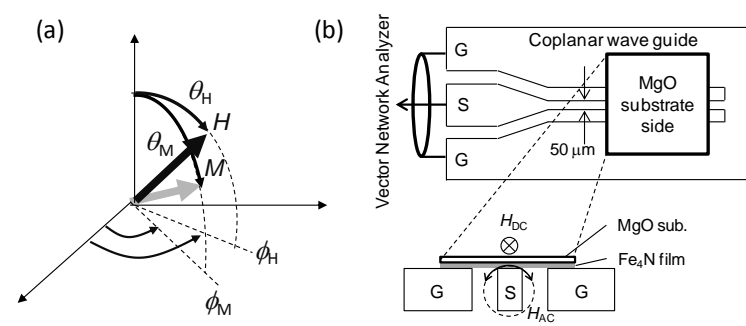

Fig. 1 (a) Definition of polar angle $(\theta)$ and azimuthal angle $(\phi)$ of external field $(H)$ and magnetization $(M)$. (b) Schematic layout of coplanar waveguide (CPW) and vector network analyzer (VNA).

optimum conditions to achieve a single-phase $\mathrm{Fe}_{4} \mathrm{~N}$ film were determined by X-ray diffraction (XRD) structural analysis, and measurements of the saturation magnetization $\left(M_{\mathrm{s}}\right)$ and negative anisotropic magnetoresistance properties. ${ }^{19)}$ Using a torque magnetometer, the in-plane magnetocrystalline anisotropy constant $\left(K_{1}\right)$ was determined using the saturated torque amplitude. The resonance field $\left(H_{\mathrm{r}}\right)$ and line-width $(\Delta H)$ under the FMR state were measured using the electron cyclotron resonance (ESR) machine with $\mathrm{X}$-band $(9.89 \mathrm{GHz})$ microwaves. Rectangular samples with dimensions of $2 \times 2 \mathrm{~mm}^{2}$ were placed on a quartz rod and introduced into the microwave cavity $\left(\mathrm{TE}_{011}\right)$. In the in-plane case, $H$ was applied along [100] and [110] of the epitaxial $\mathrm{Fe}_{4} \mathrm{~N}$ film. In the out-of-plane case, $H$ was rotated in the (010) and (110) planes of the film to evaluate $\alpha$ from $\Delta H$. The sample temperature ( $T$ ) was changed from R.T. to $4 \mathrm{~K}$. Another FMR experiment was performed as a reference measurement by scanning the applied microwave frequency using a vector network analyzer (VNA) and a coplanar waveguide (CPW). The setups are shown in Fig. 1(b).

The total free energy density of magnetization in this study is expressed as

$$
\begin{aligned}
E & =-M_{s} H\left[\sin \theta_{H} \sin \theta_{M} \cos \left(\phi_{H}-\phi_{M}\right)+\cos \theta_{H} \cos \theta_{M}\right] \\
& +\left(2 \pi M_{s}^{2}-K_{\perp}\right) \cos ^{2} \theta_{M} \\
& +\left(\frac{K_{1}}{4}\right)\left[\sin ^{4} \theta_{M} \sin ^{2} \phi_{M}+\sin ^{2} 2 \theta_{M}\right]
\end{aligned}
$$

where $M_{\mathrm{s}}, H, \quad \theta_{\mathrm{H}(\mathrm{M}),} \phi_{\mathrm{H}(\mathrm{M}),}$ and $K_{1}$ represent the saturation magnetization, external field, polar angle of the external field (magnetization) with respect to the [001] direction, azimuthal angle of the external field (magnetization) with respect to the [100] direction, and the magnetocrystalline anisotropy constant, respectively. Based on Eq. (1), the actual equation in the FMR condition is given by
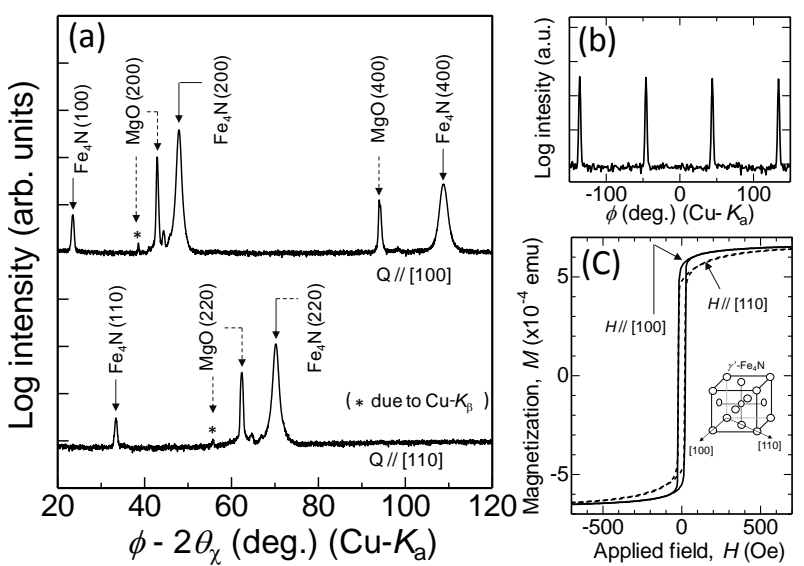

Fig. 2 (a) In-plane XRD profile of 100-nm-thick epitaxial $\mathrm{Fe}_{4} \mathrm{~N}$ film measured with $\mathrm{X}$-ray incidence angle of $0.4^{\circ}$ with respect to film surface. Upper and lower profiles were observed with scattering vector (Q) points to [100] and [110] of $\mathrm{Fe}_{4} \mathrm{~N}$, respectively. (b) $\phi$ scan profile with $2 \theta_{x}=47.8^{\circ}$, which corresponds to the $\mathrm{Fe}_{4} \mathrm{~N}(200)$ diffraction angle. (c) In-plane magnetization hysteresis loops ( $M-H$ loops) at RT with external field applied to [100] (solid line) and [110] (dashed line).

$$
\begin{aligned}
\frac{\partial E}{\partial \theta_{M}} & =0 \text { and } \\
\left(\frac{2 \pi f_{r}}{\gamma}\right)^{2} & =\left[\frac{\partial^{2} E}{\partial \phi_{M}^{2}} \frac{\partial^{2} E}{\partial \theta_{M}^{2}}-\left(\frac{\partial^{2} E}{\partial \phi_{M} \partial \theta_{M}}\right)^{2}\right] \\
& =\left[H_{r} \cos \left(\theta_{H}-\theta_{M}\right)+\mathrm{A}\right] \times\left[H_{r} \cos \left(\theta_{H}-\theta_{M}\right)+\mathrm{B}\right] .
\end{aligned}
$$

Here, $\mathrm{A}$ and $\mathrm{B}$ are functions of $\theta_{\mathrm{H}(\mathrm{M})}$ and $\phi_{\mathrm{H}(\mathrm{M}) \text {. To extract }}$ $\alpha$ from the effective magnetic damping constant $\left(\alpha_{\text {eff }}\right)$, which includes the contribution attributable to inhomogeneous magnetic properties, the $\theta_{\mathrm{H}}$ dependence of $\Delta H$ was evaluated. We assumed $\Delta H=\Delta H_{\alpha}+\Delta H_{\Delta 4} \pi_{\text {eff }}$ $+\Delta H_{\Delta \theta}+\Delta H_{\mathrm{K} 1}$, where $\Delta H_{\alpha}, \Delta H_{\Delta 4 \pi} M_{\mathrm{eff}}, \Delta H_{\Delta \theta}$, and $\Delta H_{\mathrm{K} 1}$ represent the line-width due to intrinsic magnetic damping, the distribution of the magnitude of demagnetization, the distribution of the $H$ direction, and distribution of $K_{1}$, respectively. The respective formulas are expressed as

$$
\begin{aligned}
& \Delta H_{\alpha}=\frac{1}{\sqrt{3}}\left[\alpha\left(2 H_{r} \cos \left(\theta_{H}-\theta_{M}\right)+\mathrm{A}+\mathrm{B}\right)\right]\left|\frac{d\left(2 \pi f_{r} / \gamma\right)}{d H_{r}}\right|^{-1}, \\
& \Delta H_{\Delta 4 \pi M_{\text {eff }}}=\frac{1}{\sqrt{3}}\left|\frac{d H_{r}}{d\left(4 \pi M_{e f f}\right)}\right| \Delta 4 \pi M_{e f f}, \\
& \Delta H_{\Delta \theta}=\frac{1}{\sqrt{3}}\left|\frac{d H_{r}}{d \theta_{H}}\right| \Delta \theta_{H}, \\
& \Delta H_{K_{1}}=\frac{1}{\sqrt{3}}\left|\frac{\partial H_{r}}{\partial\left(2 \pi f_{r} / \gamma\right)} \frac{\partial\left(2 \pi f_{r} / \gamma\right)}{\partial K_{1}}\right| \Delta K_{1} .
\end{aligned}
$$




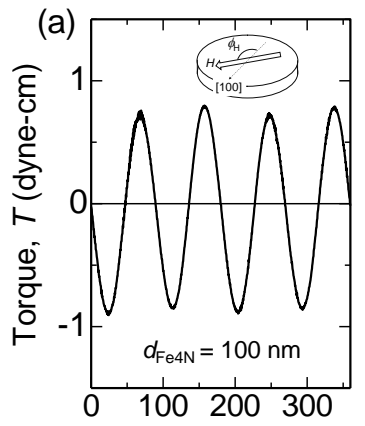

Field angle, $\phi_{\mathrm{H}}$ (deg.)

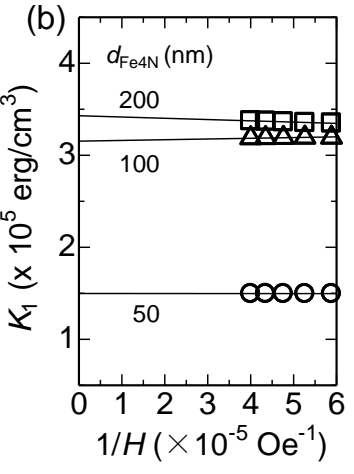

Fig. 3 (a) Torque curve of 100-nm-thick epitaxial $\mathrm{Fe}_{4} \mathrm{~N}$ film with diameter of $8 \mathrm{~mm}$. (b) Magnetocrystalline anisotropy constant $\left(K_{1}\right)$ as a function of the reciprocal of the applied field $(1 / H)$. The open circles, triangles, and squares correspond to experimentally obtained $K_{1}$ values for thicknesses of 50, 100, and $200 \mathrm{~nm}$, respectively. The solid lines plot the linear fitting results using the least-squares method.

In this study, by fitting the experimental $\Delta H$ as a function of $\theta_{\mathrm{H}}$ using Eqs. (4)-(7), $\alpha$ was evaluated.

\section{Experimental results}

\subsection{Crystallographic textures and magnetic properties of epitaxial $\mathrm{Fe}_{4} \mathrm{~N}$ thin film}

Figure 2(a) shows the XRD profile with grazing incidence, the $\phi 2 \theta_{\chi}$ scan. Both profiles for the X-ray scattering vectors $(Q)$ set to [100] and [110] of $\mathrm{Fe}_{4} \mathrm{~N}$ are shown on the same panel. Diffraction from the $\mathrm{Fe}_{4} \mathrm{~N}$ (100) and (110) superlattice appeared at $23.6^{\circ}$ and $33.5^{\circ}$, respectively. Judging from the estimation using the Bragg's formula, small diffractions that appeared at $44.3^{\circ}$ for $Q / /$ [100] and at $64.6^{\circ}$ for $Q / /$ [110] might correspond to the bcc-Fe (110) and (200), respectively. Such the speculation implies the existence of ultra-thin Fe film, an initial growth layer, with $\alpha$ phase at the interface between $\mathrm{MgO}$ substrate and $\mathrm{Fe}_{4} \mathrm{~N}$ film due to its large lattice misfit to be $10.8 \%$. The $\phi$ scan profile is shown in Fig. 2(b). Diffraction peaks every $90^{\circ}$ appeared with almost the same intensity. The results revealed that a $\mathrm{Fe}_{4} \mathrm{~N}$ film was successfully grown on the $\mathrm{MgO}(100)$ single crystal substrate. Figure 2(c) shows the in-plane magnetization hysteresis loops ( $M$ - $H$ loops) measured at R.T. with $H / /$ [100] and [110] of the $\mathrm{Fe}_{4} \mathrm{~N}$ crystal. In the specimen, magnetization saturates at $H$ $=400$ Oe and 700 Oe for [100] and [110], respectively. $M_{\mathrm{s}}$ of the epitaxial thin film was measured to be 1390 $\mathrm{emu} / \mathrm{cm}^{3}$, which agreed with that of the bulk $\mathrm{Fe}_{4} \mathrm{~N}$ (1400 $\sim 1500 \mathrm{emu} / \mathrm{cm}^{3}$ ) (e.g. Ref. 20) as well as the film (1470 emu/cm ${ }^{3}$ ) fabricated using molecular beam epitaxy. ${ }^{21}$ ) However, the $M_{\mathrm{r}} / M_{\mathrm{s}}$ ratio becomes less than 1 for [100]. That might be explained by an ultra-thin bcc-Fe initial growth layer, of which hard-axis $([110])$ is parallel to
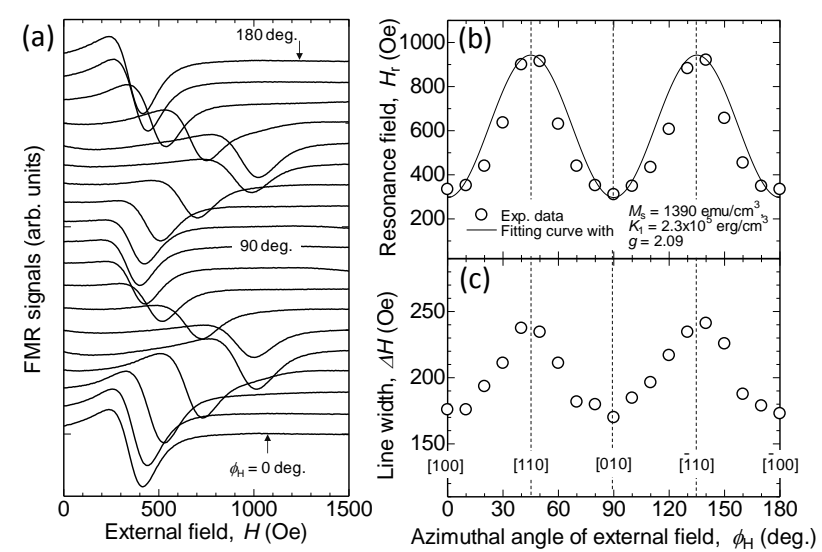

Fig. 4 (a) In-plane FMR spectra obtained by varying the azimuthal angle of the external field $\left(\phi_{\mathrm{H}}\right)$ with respect to the [100] crystallographic axis of the epitaxial $\mathrm{Fe}_{4} \mathrm{~N}$ film. (b) Resonance field $\left(H_{\mathrm{r}}\right)$ as a function of $\phi_{\mathrm{H}}$. The open circles and solid line represent experimental data and calculated results using Eq. (3) with $\theta_{\mathrm{M}}=\theta_{\mathrm{H}}=90$ deg. (c) Experimentally obtained line-width $(\Delta H)$ as a function of $\phi \mathrm{H}$.

the [100] of $\mathrm{Fe}_{4} \mathrm{~N}$.

Figure 3(a) shows the torque-curve of the 100-nm-thick $\mathrm{Fe}_{4} \mathrm{~N}$ film. $H$ was rotated in the (001) plane. A four-fold symmetric curve was observed with negligibly small uniaxial anisotropy. Judging from the curve, $<100>$ and $<110>$ correspond to the magnetization easy-axis and hard-axis, respectively, which was also confirmed by the $M-H$ loops shown in Fig. 2(c).

Figure 3(b) shows the reciprocal of $H(1 / H)$ dependence of the $K_{1}$ values evaluated using the saturated torque amplitude for 50, 100, and 200-nm-thick epitaxial $\mathrm{Fe}_{4} \mathrm{~N}$ films. The intrinsic $K_{1}$ was determined to be the value for $1 / H=0$, that is $H \rightarrow \infty$, with extrapolation of the least-squares fitting line so that domain walls could be extracted. $K_{1}$ for $50 \mathrm{~nm}$ thickness was half the values of $K_{1}$ for $100 \mathrm{~nm}$ and 200 nm thicknesses, which were similar. These results imply that the $\mathrm{Fe}_{4} \mathrm{~N}$ with highly chemical ordering dominates the film with increasing thickness, and that biaxial anisotropy might be saturated at a thickness around $100 \mathrm{~nm}$. The following FMR experiment was conducted for the 100-nm-thick $\mathrm{Fe}_{4} \mathrm{~N}$ film.

\subsection{External field dependence of in-plane FMR at R.T.}

Figure 4(a) shows the in-plane FMR spectra obtained by varying the azimuthal angle of the external field $\left(\phi_{\mathrm{H}}\right)$ with respect to the [100] for the epitaxial $\mathrm{Fe}_{4} \mathrm{~N}$ film. The spectrum indicates the magnitude of microwave power absorption by magnetization precession, and a dispersion-type spectrum is observed by $H$ modulation. From 0 to 180 deg., the spectra systematically shifted 

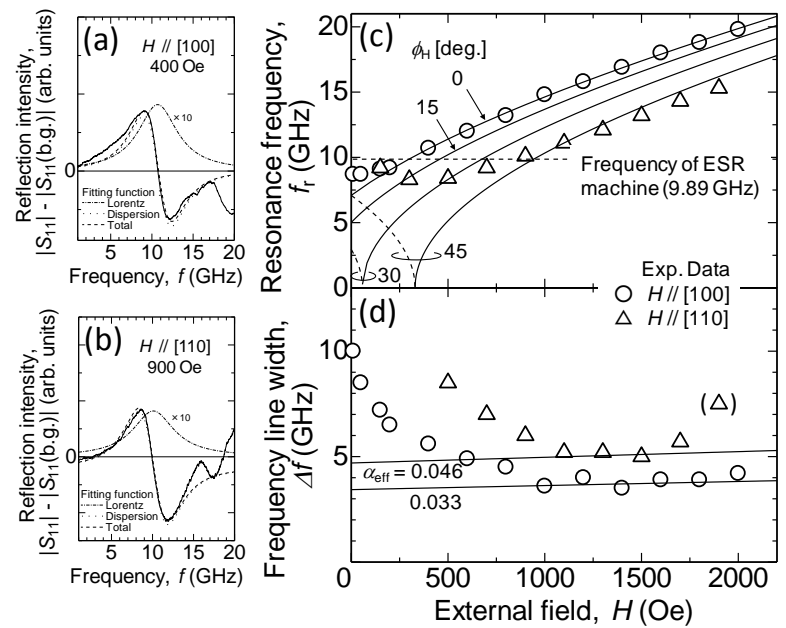

Fig. 5 Typical FMR spectra $\left(\left|S_{11}\right|\right)$ measured at R.T. using coplanar waveguide (CPW) and vector network analyzer (VNA) for (a) $H / /$ [100] and (b) $H / /$ [110]. The spectra were fitted with dispersion and Lorentz functions. (c) Resonance frequency $\left(f_{r}\right)$ as a function of $H$. The open circles and triangles correspond to experimental $f_{\mathrm{r}}$ with $H / /$ [100] and [110]. The lines are calculated $f_{\mathrm{r}}$ values obtained by varying azimuthal angle $\left(\phi_{\mathrm{H}}\right)$ with respect to [100]. (d) Frequency linewidth $(\Delta f)$ as a function of $H$. The solid lines are calculated $\Delta f$ values using the equation: $2 \pi \Delta f=2 \alpha_{\text {eff }} \gamma H_{\text {eff. }}$

\section{depending on $\phi_{\mathrm{H}}$.}

Figure 4(b) shows the $\phi_{\mathrm{H}}$ dependence of $H_{\mathrm{r}}$, which is defined as the intersection field at which the horizontal baseline of the background crosses the spectrum itself. The experimental data show that four-fold symmetric behavior such that $H_{\mathrm{r}}=910$ Oe $(310 \mathrm{Oe})$ was observed at $\left.\phi_{\mathrm{H}}=\langle 110\rangle(<100\rangle\right)$. We confirmed that a large (small) $H_{\mathrm{r}}$ was observed for the magnetic hard (easy) direction. This must imply the presence of biaxial magnetocrystalline anisotropy in the (001) plane. The FMR resonance condition in the presence of magnetocrystalline anisotropy was considered by Kittel for the case in which $H$ was applied parallel to the film plane. ${ }^{22)}$ Using Eqs. (1), (2), and (3) with $\theta_{\mathrm{M}}=\theta_{\mathrm{H}}=90$ deg. and $\phi_{\mathrm{H}}=\phi_{\mathrm{M}}$, the modified FMR relationship is given by

$\left(\frac{2 \pi f_{r}}{\gamma}\right)^{2}=\left[H_{r}+\frac{K_{1}}{M_{s}}\left(2-\sin ^{2} 2 \phi_{M}\right)+4 \pi M_{s}\right]\left[H_{r}+\frac{2 K_{1}}{M_{s}} \cos 4 \phi_{M}\right]$, (8)

where $f_{\mathrm{r}}$ and $\gamma$ represent the resonance frequency of 9.89 $\mathrm{GHz}$ and the gyromagnetic constant in CGS units, $\gamma=$ $g e / 2 m_{\mathrm{e}} c=17.588 \times g \mathrm{MHz} / \mathrm{Oe}$, respectively. Here, we assumed that the perpendicular anisotropy was omitted $\left(4 \pi M_{\text {eff }}=4 \pi M_{\mathrm{s}}-2 K_{\downarrow} / M_{\mathrm{s}} \approx 4 \pi M_{\mathrm{s}}\right)$ because our specimen had sufficiently large $M_{\mathrm{s}}$ compared to $K_{\perp}$. With $M_{\mathrm{s}}=$ $1390 \mathrm{emu} / \mathrm{cm}^{3}, K_{1}=2.3 \times 10^{5} \mathrm{erg} / \mathrm{cm}^{3}$ and $g=2.09$, the calculation showed good agreement with the maximum and minimum $H_{\mathrm{r}}$ of the experimental data. $M_{\mathrm{s}}$ and $K_{1}$ had similar values as observed in the $M-H$ loop and the torque-curve as mentioned above. The slight difference in intermediate $H_{\mathrm{r}}$ might be due to a slightly different azimuthal angle between $\phi_{\mathrm{H}}$ and $\phi_{\mathrm{M}}$.

Figure 4(c) shows the $\phi_{\mathrm{H}}$ dependence of $\Delta H . \Delta H$ is defined as the field difference at positive and negative FMR peaks. Likely to the $H_{\mathrm{r}}$ behavior, the experimental data show four-fold symmetric properties; $\Delta H=240 \mathrm{Oe}$ $\left(175\right.$ Oe) was observed at $\phi_{\mathrm{H}}=<110>\quad(<100>)$. Converting $\Delta H$ to $\alpha_{\text {eff }}$ using the Gilbert damping equation: $\Delta H=(2 / \sqrt{ } 3) \alpha(2 \pi f) / g$, $\alpha_{\text {eff }}$ was estimated to be 0.062 and 0.045 for $<110>$ and $<100>$, respectively.

\subsection{Frequency dependence of in-plane FMR via CPW and VNA at R.T.}

To confirm anisotropic $\alpha_{\text {eff }}$ in the epitaxial $\mathrm{Fe}_{4} \mathrm{~N}$ film observed using ESR, $\alpha_{\text {eff }}$ in the film plane was also investigated by means of CPW and VNA by scanning the microwave frequency. Figures 5(a) and 5(b) show the reflection intensity spectra $\left(\left|S_{11}\right|\right)$ as a function of the applied frequency $(f)$ with $H / /$ [100] and $H / /$ [110], respectively. Since $\left|S_{11}\right|$ included the reflection component attributable to the CPW itself as background (| $S_{11}$ (b.g.)|), after applying sufficiently large $H$ in the film plane so that spectrum attributable to FMR component disappears, $\mid S_{11}$ (b.g.)| was subtracted from $\left|S_{11}\right|$. The imaginary part of $S_{11}$, corresponding to the phase-lag from the input power, dominated the spectra in our specimen. For this reason, in order to determine the resonance frequency $\left(f_{\mathrm{r}}\right)$ and the frequency line-width $(\Delta f)$, we performed fitting using the following Lorentz and its derivative functions, namely, the dispersion function with proportional constants $(\mathrm{P}, \mathrm{Q})$.

$$
\left|S_{11}\right|=\mathrm{P} \frac{\Delta f}{\left(f-f_{r}\right)^{2}+\Delta f^{2}}+\mathrm{Q} \frac{\Delta f\left(f-f_{r}\right)}{\left[\left(f-f_{r}\right)^{2}+\Delta f^{2}\right]^{2}} .
$$

Both of the experimental spectra were reproduced by Eq. (9). Though the dip signals appeared at $f=20 \mathrm{GHz}$ for $H$ // [100] and $f=17 \mathrm{GHz}$ for $H / /$ [110], this might be attributed to the CPW and not the FMR component, since we confirmed that $f_{\mathrm{r}}$ of the dips were independent of $H$ in the experiments.

Figure 5(c) shows a summary of $f_{\mathrm{r}}$ as a function of $H$, the directions of which were $H / /$ [100] and [110]. In the case of $H / /$ [100], $f_{\mathrm{r}}$ monotonically increased from $f_{\mathrm{r}}=$ $7.5 \mathrm{GHz}$ to $20 \mathrm{GHz}$ with increasing $H$. On the other hand, in the case of $H / /$ [110], after the parabolic dependence appeared up to $H=750 \mathrm{Oe}$, a monotonic increase appeared. The relationship between $f_{\mathrm{r}}$ and $H$ in the specimen is given by Eq. (8). The solid lines show the calculation results for $\phi_{\mathrm{H}}=0(H / /[100]), 15,30$, and $45 \mathrm{deg}$. $(H / /[110])$. The same parameters including $M_{\mathrm{s}}$ $=1390 \mathrm{emu} / \mathrm{cm}^{3}, K_{1}=2.3 \times 10^{5} \mathrm{erg} / \mathrm{cm}^{3}$ and $g=2.09$ are substituted. However, Eq. (8) is valid only when $H$ is greater than $H_{\mathrm{K} 1}=\left(2 K_{1} / M_{\mathrm{s}}\right) \cos 4 \phi \mathrm{M}$, in which case the 

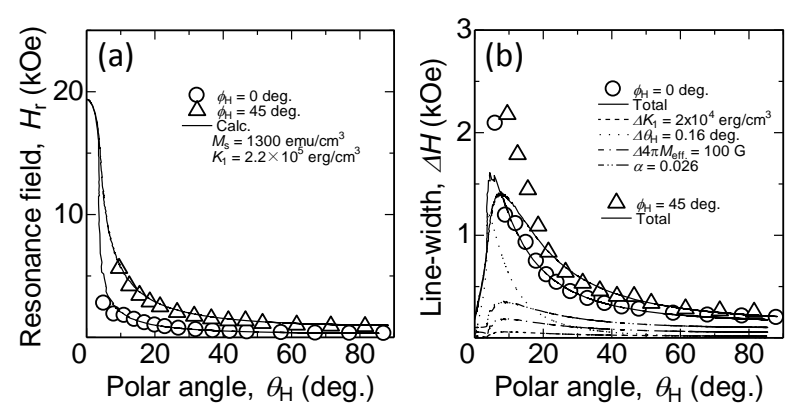

(c)

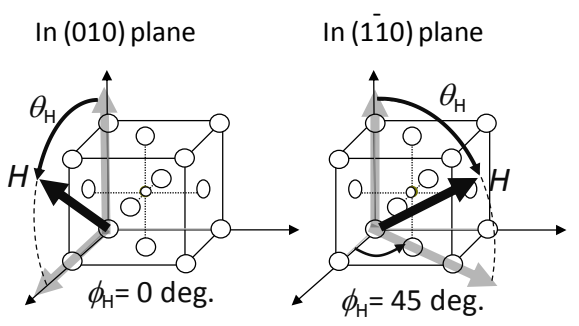

Fig. 6 (a) Resonance field $\left(H_{\mathrm{r}}\right)$ and (b) linewidth $(\Delta H)$ of the out-of-plane FMR as a function of the polar external field angle $\left(\theta_{\mathrm{H}}\right)$ with respect to [001]. Each line represents the calculated results with the parameters indicated in the panels. The open circles and triangles correspond to experimental data for $\phi_{\mathrm{H}}=0$ and 45 deg., respectively, as schematically shown in (c).

magnetization direction can maintain $\phi_{\mathrm{M}}=\phi_{\mathrm{H}}$. Modifying Eq. (8), the FMR condition formula below $H_{\mathrm{K} 1}$ is given by

$\left(\frac{2 \pi f_{r}}{\gamma}\right)^{2}=\left[-H_{r}+\frac{K_{1}}{M_{s}}\left(2-\sin ^{2} 2 \phi_{M}\right)+4 \pi M_{s}\right]\left[-H_{r}+\left|\frac{2 K_{1}}{M_{s}} \cos 4 \phi_{M}\right|\right]$,

and this result is shown by the dashed lines in Fig. 5(c). The same value of $f_{\mathrm{r}}$ can be obtained for $H=0$ Oe, which means the FMR is achieved by $H_{\mathrm{K} 1}$ pointing to [100], easy-axis. The calculation revealed that the experimental $f_{\mathrm{r}}$ for $H / /$ [100] was fitted well with $\phi_{\mathrm{H}}=0$ for all $H$. In the case of $H / /$ [110], only $f_{\mathrm{r}}$ above $H=900$ Oe could be reproduced by the calculation. Below 900 Oe, it is possible to interpret this as $\phi_{\mathrm{M}}$ gradually changing from 45 to 0 deg. because of $H_{\mathrm{K} 1}$ with decreasing $H$, and finally reaching complete 0 deg. ([100] easy-axis) at $H=0$, which means that FMR condition is achieved by only $H_{\mathrm{K} 1}$ at $H=0$. Note that $H_{\mathrm{r}}$ $=900 \mathrm{Oe}(250 \mathrm{Oe})$ was observed at $f=9.89 \mathrm{GHz}$, which was consistent with the $H_{\mathrm{r}}$ obtained by ESR.

Figure 5(d) shows $\Delta f$ as a function of $H$, the direction of which is $H / /$ [100] and [110]. $\Delta f$ is defined as the FWHM of the Lorentz function expressed by Eq. (9) after fitting the spectra. We can confirm that a large $\Delta f$ for [110] was observed for all values of $H$. Here, $\alpha_{\text {eff }}$ was evaluated using the simple FMR equation: $2 \pi \Delta f=$ $2 \alpha_{\text {eff }} \gamma H_{\text {eff, }}$ where $H_{\text {eff }}$ includes $H, 4 \pi M_{\mathrm{s}}$, and $H_{\mathrm{K} 1}$. By fitting the experimental data where a constant $\Delta f$ was observed in the high $H$ region, $\alpha_{\text {eff }}$ for [100] and [110] were determined to be 0.033 and 0.046 , respectively, with $M_{\mathrm{s}}=1390 \mathrm{emu} / \mathrm{cm}^{3}, K_{1}=2.3 \times 10^{5} \mathrm{erg} / \mathrm{cm}^{3}$, and $g=$ 2.09. Consistent with the data obtained by ESR, $\alpha_{\text {eff }}$ of [100] is obviously larger than that of [110] in our specimen at R.T.

\subsection{Anisotropic intrinsic damping constant and its temperature dependence}

In order to extract $\alpha$ from $\alpha_{\text {eff, }}$ experiments on out-of-plane FMR were conducted. Figure 6(a) shows the $\theta_{\mathrm{H}}$ dependence of $H_{\mathrm{r}}$ at R.T. when $H$ rotates in the (010) plane $\left(\phi_{\mathrm{H}}=0\right.$ deg.) and the (110) plane $\left(\phi_{\mathrm{H}}=45\right.$ deg.), respectively. Because of the $H$ limitation of our ESR machine, data below $\theta_{\mathrm{H}}=5 \mathrm{deg}$ are not shown. Both $H_{\mathrm{r}}$ increased with decreasing $\theta_{\mathrm{H}}$ due to the demagnetization field generated perpendicular to the film plane. $H_{\mathrm{r}}$ was enhanced for $\phi_{\mathrm{H}}=45$ deg. compared to $H_{\mathrm{r}}$ for $\phi_{\mathrm{H}}=0$ deg. for all $\theta_{\mathrm{H}}$. By substituting $\phi_{\mathrm{H}}=0$ and 45 deg. in the modified Eq. (3), the calculation was conducted with $M_{\mathrm{s}}=1300 \mathrm{emu} / \mathrm{cm}^{3}, K_{1}=2.2 \times 10^{5}$ $\mathrm{erg} / \mathrm{cm}^{3}$ and $g=2.09$. The parameters were consistent with values obtained in the in-plane FMR. The calculations reveal that the difference in $H_{\mathrm{r}}$ must be due to the presence of $K_{1}$.

Figure 6(b) shows the $\theta_{\mathrm{H}}$ dependence of $\Delta H$ at R.T. for $\phi_{\mathrm{H}}=0$ and $45 \mathrm{deg}$. The calculation of $\Delta H$ was also conducted assuming the extrinsic contributions mentioned in Eqs. (4), (5), (6), and (7). Except for the less than $\phi_{\mathrm{H}}=20 \mathrm{deg}$. in the $\phi_{\mathrm{H}}=45 \mathrm{deg}$. case $(7 \mathrm{deg}$. in the $\phi_{\mathrm{H}}=0$ deg. case), we found that the calculations agreed with experimental data. In prior research on out-of-plane FMR in $\mathrm{Ni}-\mathrm{Fe}, \mathrm{Co}-\mathrm{Fe}-\mathrm{B}, \mathrm{Co}_{2} \mathrm{MnSi}$, and even poly-crystalline $\mathrm{Fe}_{4} \mathrm{~N}$, the whole $\Delta H$ was successfully fitted by calculation.6,18,23-26) Even though the mechanism is not yet fully identified, judging from the fact that the unmatched fitting result appears stronger for $\phi_{\mathrm{H}}=45$ deg., domain structures in small $\theta_{\mathrm{H}}$ are considered as a possible mechanism because of $K_{1}$ in the epitaxial $\mathrm{Fe}_{4} \mathrm{~N}$ film. Such an additional resonance has been presumed to be domain structure excitation below magnetic saturation in crystals with strong magnetic anisotropy. ${ }^{27,28)}$ For example, Nagamiya examined the additional resonance observed in $\mathrm{Fe}_{3} \mathrm{O}_{4}$ where the magnetic anisotropy has tetragonal symmetry. ${ }^{29)}$ Therefore, we performed the fitting for the large $\theta_{\mathrm{H}}$ region, where a single domain structure could be expected.

Figure 7 shows the temperature dependence of $\alpha$ determined by data fitting of the out-of-plane FMR experiments mentioned above for $\phi_{\mathrm{H}}=0$ and $45 \mathrm{deg}$. The error bars attached to each data point show the value of $2 \sigma$ assuming that our experiments and analysis events follow the normal distribution. We can confirm that the distributions of magnetic-properties-related extrinsic damping components such as $\Delta 4 \pi M_{\text {eff, }}, \Delta \theta_{\mathrm{H}}$, 


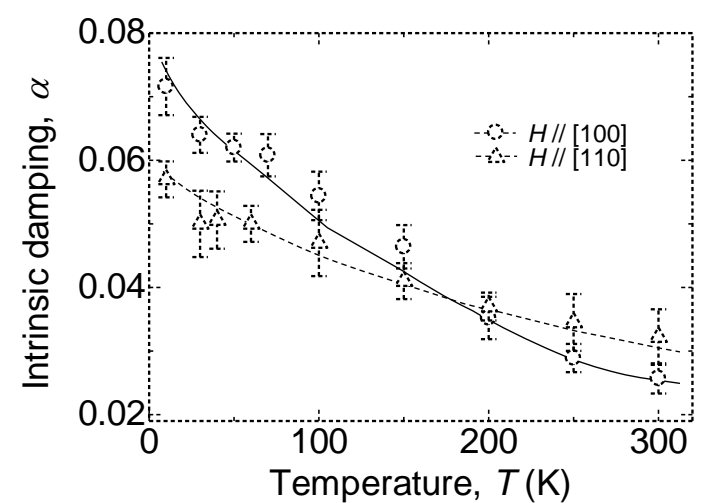

Fig. 7 Temperature dependence of the intrinsic magnetic damping constant $(\alpha)$ for $\phi_{\mathrm{H}}=0(H / /[100])$ and 45 deg. ( $H / /[110])$. Error bars $(2 \sigma)$ have been included. Solid and dashed lines are visual guides.

and $\Delta K_{1}$ were successfully excluded, therefore $\alpha$ reflects the damping phenomena for our material itself. Note that anisotropic $\alpha$ was clearly observed at R.T., that is, $\alpha$ for $\phi_{\mathrm{H}}=45 \mathrm{deg}$. was larger than that for $\phi_{\mathrm{H}}=0 \mathrm{deg}$., and both $\alpha$ were enhanced with decreasing $T$. Surprisingly, $\alpha$ for $\phi_{\mathrm{H}}=0 \mathrm{deg}$. exceeded that for $\phi_{\mathrm{H}}=45$ deg. at $T \approx 180 \mathrm{~K}$. The phenomenon has not yet been reported. To understand the principles such as $T \approx 180$ $\mathrm{K}$ as well as the magnitude relation between $\alpha$ for [100] and [110], the first principle numerical calculation assuming spin-orbit interaction of $\mathrm{Fe}_{4} \mathrm{~N}$ is indispensable.

\section{Discussion}

Anisotropic $\alpha$, in other words, different $\alpha$ depending on the magnetization direction with respect to the lattice, was clearly observed in the epitaxial $\mathrm{Fe}_{4} \mathrm{~N}$ film. To discuss the mechanism for the occurrence of anisotropic $\alpha$, we examined the numerical calculation of $\alpha$ for the $\mathrm{Fe}_{4} \mathrm{~N}$ crystalline model (bulk) based on the torque correlation model developed by Kambersky and Gilmore et al. ${ }^{16,17)}$ Figure. 8 shows the calculation result conducted for both cases in which the magnetization direction points along [100] and [110] using the linear-muffin-tin orbital (LMTO) method under the local spin density functional approximation (LSDA). ${ }^{30}$ The actual form of $\alpha$ in our calculation is

$\alpha \propto \sum_{k} \sum_{m, n}\left(\left|\Gamma_{m k, n k}\right|^{2} \frac{\hbar / 2 \tau}{\left(E_{F}-E_{m k}\right)^{2}+(\hbar / 2 \tau)^{2}} \frac{\hbar / 2 \tau}{\left(E_{F}-E_{n k}\right)^{2}+(\hbar / 2 \tau)^{2}}\right)$ (11) where $\Gamma_{\mathrm{mk}, \mathrm{nk}}, E_{\mathrm{F}}$, and $E_{\mathrm{mk}(\mathrm{nk})}$ represent the matrix element of the torque due to spin-orbit interaction, the Fermi energy, and the electron energy of the $m(n)$ band at $k$ space, respectively. Since $\tau$ represents the electron lifetime attributable to the electron-lattice interaction, $1 / \tau$ of the lateral axis indicates temperature. We can find two facts from the calculation results: first, both $\alpha$ decrease exponentially with increasing temperature;

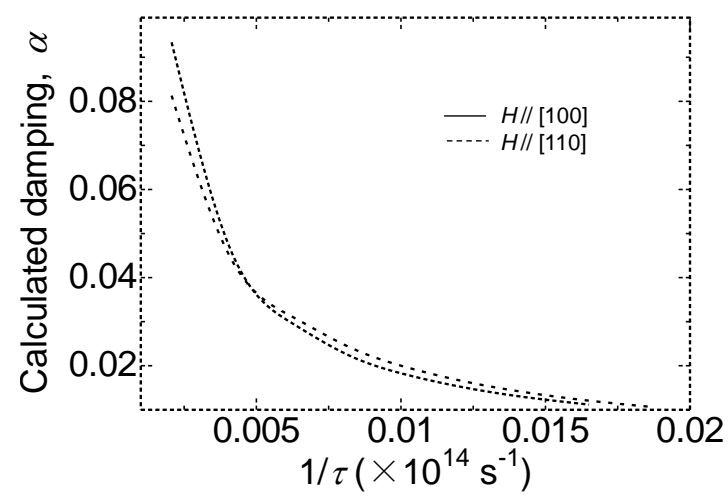

Fig. 8 Numerical calculation of intrinsic damping constant $(\alpha)$ as a function of the inverse of electron lifetime $(1 / \tau)$ of the epitaxial $\mathrm{Fe}_{4} \mathrm{~N}$. The solid and dashed lines correspond to the results when the external field $(H)$ was applied along [100] and [110].

second, $\alpha$ for $H / /$ [110] is larger than $\alpha$ for $H / /$ [100] above $1 / \tau \approx 0.005 \times 10^{14} \mathrm{~s}^{-1}$, although its magnitude relation is reversed below $1 / \tau$. The interpretation for the first result is that the monotonic decrease with $1 / \tau$ indicates that the events of excitation and relaxation of electrons take place within the same band $(m=n)$, corresponding to the "intra-band" transition. The second result can be explained by considering that Eq. (11) is constituted of two Lorentzian spectra with energy broadening of $1 / \tau$. Therefore, if the states connected by the matrix elements $\Gamma_{\mathrm{mk}, \mathrm{mk}}$ are sharply distributed around the $E_{\mathrm{F}}$, a small value of $1 / \tau$ is superior for $\alpha$, and then we infer that this may be the case for $H / /[100]$ situation below $1 / \tau \approx 0.005 \times 10^{14} \mathrm{~s}^{-1}$. Contrarily, in the case of $H / /$ [110], it is considered that the energy distribution of the state crucial for $\Gamma_{\mathrm{mk}, \mathrm{mk}}$ is wide compared to the $H / /$ [100] case. In the presence of spin-orbit interaction, the electron band structure changes depending on the magnetization direction and then the effects of energy broadening $1 / \tau$ on the excitation spectra may be different. Based on our calculations, the results lead us to conclude that the experimental anisotropic $\alpha$ and its $T$ dependence reflect the difference in electron band structures of $\mathrm{Fe}_{4} \mathrm{~N}$ depending on $H$ and $T$.

However, $T$ at which $\alpha$ for $H / /$ [100] exceeds that for $H / /$ [110] (crossover T) comes out to be extremely low in the calculation. In the electrical conduction model, resistivity $(\rho)$ is generally given by

$$
\rho=\frac{m_{e}}{n e^{2}} \frac{1}{\tau},
$$

where $m_{\mathrm{e}}, n$, and $e$ represent the electron mass, the free-electron density, and the elementary charge, respectively. Using the formula, $1 / \tau \approx 0.005 \times 10^{14} \mathrm{~s}^{-1}$ is converted to $\rho=0.018 \mu \Omega \mathrm{cm}$ with $m_{\mathrm{e}}=9.1 \times 10^{-31} \mathrm{~kg}, n$ $=1 \times 10^{29} \mathrm{~m}^{-3}$, and $e=1.6 \times 10^{-19} \mathrm{C}$. According to the experimental data for the $T$ dependence of $\rho$ in the epitaxial $\mathrm{Fe}_{4} \mathrm{~N}$ film, ${ }^{31)} \rho=0.018 \mu \Omega \mathrm{cm}$ cannot be 
explained within the finite $T$ regime since even the residual $\rho$ is 100 times as high as the converted $\rho=$ $0.018 \mu \Omega \mathrm{cm}$. Though further investigation is warranted, in our speculation, the difference in temperature might be attributed to the actual $\tau$ of the $\mathrm{Fe}_{4} \mathrm{~N}$ film possibly being extremely short due to scattering by impurities and/or dislocations in the thin films.

\section{Summary}

Studies on in-plane FMR and out-of-plane FMR were carried out for 100-nm-thick epitaxial $\mathrm{Fe}_{4} \mathrm{~N}$ films. Large (small) $H_{\mathrm{r}}$ and $\Delta H$ were observed for $H / /$ [110]: hard-axis ( $H / /$ [100]: easy-axis). Calculation of $H_{\mathrm{r}}$ using a modified Kittel's function agreed with the experimental $H_{\mathrm{r}}$ with reasonable parameters: $M_{\mathrm{s}}=1390$ $\mathrm{emu} / \mathrm{cm}^{3}, K_{1}=2.3 \times 10^{5} \mathrm{erg} / \mathrm{cm}^{3}$ and $g=2.09$. As a comparison, in-plane FMR using CPW and VNA was performed at R.T., and it was confirmed that the above three parameters were also valid to explain the experimental data. Based on the in-plane FMR study at R.T., the presence of anisotropic $\alpha$ was reliably observed in the epitaxial $\mathrm{Fe}_{4} \mathrm{~N}$ film. Using the out-of-plane FMR, temperature variation of the anisotropic $\alpha$ was investigated. We found that $\alpha$ for $\phi_{\mathrm{H}}=0$ deg. was significantly enhanced with decreasing $T$, and exceeded that for $\phi_{\mathrm{H}}=45 \mathrm{deg}$. at $T=180 \mathrm{~K}$. According to our numerical calculation of $\alpha$ as a function of $1 / \tau$, the observed anisotropic $\alpha$ was at least qualitatively explained by the change in the electronic band structure depending on the magnetization direction.

Acknowledgements The authors appreciate Dr. M. Mizuguchi in Institute for Materials Research, Tohoku University, for measurements of FMR with CPW and VNA. This work has been supported by a Grant-in-Aid for Scientific Research from the JSPS, KAKENHI Grant Number 24710154.

\section{References}

1) S. Kokado, N. Fujiwara, K. Harigaya, H. Shimizu and A. Sakuma: Phys. Rev. B, 73, 172410 (2006).

2) K. Sunaga, M. Tsunoda, K. Komagaki, Y. Uehara and M. Takahashi: J. Appl. Phys., 102, 013917 (2007).

3) Y. Komasaki, M. Tsunoda, S. Isogami and M. Takahashi: J. Appl. Phys., 105, 07C928 (2009).
4) Y. Komasaki, M. Tsunoda, S. Isogami and M. Takahashi: J. Magn. Soc. Jpn., 34, 524 (2010).

5) S. Isogami, M. Tsunoda, Y. Komasaki, A. Sakuma and M. Takahashi: Appl. Phys. Express, 3, 103002 (2010).

6) R. Yilgin, Y. Sakuraba, M. Oogane, S. Mizukami, Y. Ando and T. Miyazaki: Jpn. J. Appl. Phys., 46, L205 (2007).

7) M. Sakamoto, H. Ohashi, N. Inaba, M. Ohtake, M. Futamoto and F. Kirino: The Int. Magn. Conf., Taipei, CR-12 (2011).

8) B. Aktas, B. Heinrich, G. Woltersdorf, R. Urban, L. R Tagirov, F. Yilidiz, K. Ozdogan, M. Ozdemir, O. Yalcin and B. Z. Rameev: J. Appl. Phys., 102, 013912 (2007).

9) K. Kobayashi, N. Inaba, N. Fujii, Y. Sudo, T. Tanaka, M. Ohtate, M. Futamoto and F. Kirino: IEEE Trans. on Magn., 45, 2541 (2541).

10) Y. Takahashi, H. Ikeda, N. Inaba, F. Kirino, M. Ohtate and M. Futamoto: IEEE Trans. on Magn., 47, 4682 (2011).

11) R. J. Elliott: Phys. Rev. 96, 266 (1954).

12) H. Kohno, G. Tatara and J. Shibata: J. Phys. Soc. Jpn., 75, 113706 (2006).

13) H. J. Skadsem, Y. Tserkovnyak, A. Brataas and G. E. W. Bauer: Phys. Rev. B, 75, 094416 (2007).

14) Y. Yafet: Solid State Phys., 14, 1 (1963).

15) S. M. Bhagat and P. Lubitz: Phys. Rev. B, 10, 179 (1974).

16) V. Kambersky: Phys. Rev. B, 76, 134416 (2007).

17) K. Gilmore, Y. U. Idzerda and M. D. Stiles: Phys. Rev. Lett., 99, 027204 (2007).

18) S. Isogami, M. Tsunoda, M. Oogane, A. Sakuma and M. Takahashi: Jpn. J. Appl. Phys., 52, 073001 (2013).

19) M. Tsunoda, Y. Komasaki, S. Kokado, S. Isogami, C. C. Chen and M. Takahashi: Appl. Phys. Express, 2, 083001 (2009).

20) B. C. Frazer: Phys. Rev., 112, 751 (1958).

21) K. Ito, G. H. Lee and T. Suemasu: J. Phys. Conf. Seri., 266, 012091 (2011).

22) C. Kittel: Phys. Rev., 73, 155 (1948).

23) S. Mizukami, Y. Ando and T. Miyazaki: J. Magn. Soc. Jpn., 24, 535 (2000).

24) S. Mizukami, Y. Ando, and T. Miyazaki: Jpn. J. Appl. Phys., 40, 580 (2001).

25) M. Oogane, T. Wakitani, S. Yakata, R. Yilgin, Y. Ando, A. Sakuma and T. Miyazaki: Jpn. J. Appl. Phys., 45, 3889 (2006).

26) M. Oogane, M. Watanabe, S. Yakata, Y. Ando and T. Miyazaki: J. Magn. Soc. Jpn., 31, 209 (2007).

27) J. Artman: Phys. Rev., 100, 1243 (1955).

28) H. Suhl: Phys. Rev., 97, 555 (1955).

29) T. Nagamiya: Prog. Theo. Phys., 7, 72 (1953).

30) A. Sakuma: J. Phys. Soc. Jpn., 81, 084701 (2012).

31) M. Tsunoda, H. Takahashi, S. Kokado, Y. Komasaki, A. Sakuma and M. Takahashi: Appl. Phys. Express, 3, 113003 (2010).

Received Mar. 11, 2014; Accepted May 22, 2014 